\title{
GENETIC BEHAVIOR OF SOME ROOT AND GRAIN QUALITY TRAITS UNDER WATER DEFICIT AND NORMAL CONDITIONS IN RICE (ORYZA SATIVA L.)
}

\author{
H. M. Hassan \\ Rice Research Section, Field Crops Research Institute, ARC, Giza, Egypt. \\ Received: Dec. 13, 2016 \\ Accepted: Dec. 29,2016
}

\begin{abstract}
Four rice genotypes differed in drought tolerance were crossed. Six populations (P1, P2, F1, F2, BC1 and BC2) of two rice crosses namely; Sakha 102 x IR 83142-B-60-B(cross I) and Giza $159 \times$ Takanari 1 (cross II) were raised in a randomized complete block design during the three successive summer seasons from 2014 to 2016 at the farm of Rice Research Agricultural station, Sakha, Kafr El-Sheikh, Egypt. This study aimed to determine heterosis, gene action, heritability, genetic advance and phenotypic correlation response to select and predict by the new lines for some root traits (Root length, Root volume, Number of roots/plant and Root/Shoot ratio \%) and some grain quality traits. Flush water irrigation was added every 12 days intervals. The results indicated that highly significant and positive heterosis as a deviation from mid-and better-parent were obtained for all root and grain quality, except for grain shape in the first cross and for grain length in the second cross which showed highly significant and negative estimates of heterosis as a deviation from mid-parent. In addition, incomplete dominance to over-dominance was operative for most of the studied traits.

Additive gene effect (d) and dominance gene effect ( $h$ ) were more important in the genetic system for all the studied characters, additive $x$ additive gene effects (i), additive $x$ dominance (j) and dominance $x$ dominance (I) gene effects were involved in the genetic control of all characters, except some exceptions. Heritability in broad sense was high in most traits of the two studied crosses, except cross I for root/ shoot ratio under normal condition. The highest value of heritability estimates (95.95\%) was recorded for root volume in the cross I under normal condition. The narrow sense heritability was moderate to low in most traits of the two crosses. High values of predicted genetic advance were estimated for most traits of the studied traits. Significant or highly significant positive phenotypic correlation was found between most of the studied characters for the two studied crosses especially between root and grain quality characters with grain yield/ plant, except amylose content trait.

In general the cross Giza 159 x Takanari 1 could be grown under water deficit for further screening and selecting desirable root and grain quality traits at the same time.
\end{abstract}

Key words: Rice crosses, genetic parameters, root and grain quality traits, drought tolerance.

\section{INTRODUCTION}

In Egypt, annually more than one and half million feddans are cultivated with rice, producing about 6.5 million tons of rice, with an average of 4.2 tons/ fed, (10 tons/ ha.) RRTC (2013). This average ranked at the first among the rice producing countries in the world. This production meet the needs of local consumption, and the rest is exported abroad. But, with the expected increase of population, the production should be increased.

Global climate change is expected to increase the occurrence and severity of drought episodes due to increasing temperatures and evapotranspiration. Therefore, food security in the twenty-first century will increasingly depend on the release of new cultivars with improved adaptation to drought conditions. However, selection for drought tolerance is difficult due 
to a complex genotype by environment interactions.

Drought is a major abiotic stress limiting rice production in the world. About $30 \%$ of the world's rice producing areas suffer from moisture stress and water deficit, in both rainfed and irrigated areas. About 18 million tons of rice valued at US $\$ 650$ million is lost annually due to drought, Pandey et al. (2005). For this reason, breeding for drought tolerance become of high priority in rice breeding program, especially in Egypt because of the limited irrigation water available in the River Nile. Some rice planted areas, especially those located at terminal of irrigation canals in the northern part of the Nile Delta suffer from shortage in irrigation water during different growth stages, which are considered to be one of the most serious constraints to rice production Abd Allah (2009).

In addition, after the relative success of the Green Revolution, food security has consistently been challenged by (i) population growth, (ii) urbanisation, and (iii) climate change. It is therefore now essential, not only to grow more high quality rice per hectare, but also to equip these varieties with tolerance to environmental stresses Brar and Khush (2013). To this end, significant investment has been made in many countries to improve yield and stress tolerance, while retaining quality Singh et al. (2000); Inthapanya et al. (2006); Mackill et al. (2006); Tomita (2009) and Boualaphanh et al. (2011). The current tools of quality evaluation are not sophisticated enough to define the quality each market requires, let alone enable selection for it.

The present investigation aimed to determine heterosis, degree of dominance, genetic variance, heritability, genetic advance and phenotypic correlation coefficient among some root and grain quality characters under water deficit conditions.

\section{MATERIALS AND METHODS}

The present investigation was carried out at Sakha Agricultural Research station Farm, Sakha, Kafr El-Sheikh, Egypt, during 2014, 2015 and 2016 seasons to study the genetic behavior of some root and grain quality traits in rice under water deficit and normal conditions, i.e., root length $(\mathrm{cm})$, root volume $\left(\mathrm{cm}^{3}\right)$, number of roots/ plant, root/ shoot ratio, grain length $(\mathrm{mm})$, grain shape (mm), hulling $\%$, milling $\%$, head rice $\%$ and amylose content \%.

According to the obtained results the four genotypes were crossed to produce $F_{1}$ hybrid seeds of two crosses namely; I Sakha 102 (sensitive) x IR 83142-B-60-B(tolerant). II - Giza 159 (moderate) $\mathrm{X}$ Takanari 1 (tolerant). Six populations, i.e., $\mathrm{P}_{1}, \mathrm{P}_{2}, \mathrm{~F}_{1}, \mathrm{~F}_{2}, \mathrm{BC}_{1}$ and $\mathrm{BC}_{2}$ of each cross were obtained and utilized in this study.

In 2014 season, the four parental cultivars were grown in three successive dates of planting with fifteen days interval in order to overcome the differences in flowering time between parents. Thirty days old seedlings of each parent were individually transplanted in the permanent field in seven rows. Each row was $5 \mathrm{~m}$ long and contained 25 hills spaced $20 \mathrm{~cm}$ apart. At flowering time, hybridization between parents was carried out following the technique proposed by Jodon (1938) and modified by Butany (1961). And the aforementioned two crosses were produced.

In 2015 season, parents and $F_{1}$ hybrid seeds of the two crosses together with their parental lines were planted under normal conditions. At heading, parents were crossed again to produce the $F_{1}$ hybrid seeds of the two crosses following the same technique. Moreover, some of $F_{1}$ plants were left to be self pollinated in order to produce $F_{2}$ seeds, while some other $F_{1}$ plants were crossed with their own parents to produce $\mathrm{BC}_{1}$ and $\mathrm{BC}_{2}$ seeds. At harvest, seeds of different generations were individually harvested to be grown in the next season. Subsequently, in the summer 
season of 2016, seeds of $P_{1}, P_{2}, F_{1}, B C_{1}$, $\mathrm{BC}_{2}$ and $\mathrm{F}_{2}$ of each cross were sown under drought conditions. Six population of each cross (parents, $F_{1 ' s}, F_{2 ' s}, B_{1}$, and $B C 2$ ) were planted in a randomized complete block design experiment with three replications. Each replicate contained 10 rows of each of $\mathrm{P}_{1}, \mathrm{P}_{2}$ and 5 rows of each of $\mathrm{F}_{1}, \mathrm{BC}_{1}$ and $\mathrm{BC}_{2}$ and 20 rows of $\mathrm{F}_{2}$. Rows were $5 \mathrm{~m}$ long and $20 \times 20 \mathrm{~cm}$ apart. In all growing seasons of the study, all cultural practices were applied as recommended. The six populations in 2016 season were planted under water deficit conditions (water deficit was imposed by using flush irrigation every 12 days without standing water after irrigation). Hand weeding was done when it was needed. Sixty plants from each $P_{1}, P_{2}$ and $F_{1}, 90$ plants from each $\mathrm{BC}_{1}$ and $\mathrm{BC}_{2}$ and 200 plants from each $F_{2}$ populations were taken at random. These plants were individually harvested and threshed separately to determine the grain yield/plants and yield components.

Heterosis was estimated according to Falconer and Mackay (1996). Furthermore, appropriate L. S. D. values were calculated to test the significance of heterotic effects according to the formula suggested by Wynne et al. (1970). The relative potence ratio $(P)$ was used to determine the degree of dominance and its directions according to the formula given by Mather and Jinks (1971). Estimation of gene effects were suggested by Mather (1949) and Hayman (1958). Expected genetic variances of $\mathrm{VBC}_{1}$, $\mathrm{VBC}_{2}$ and $\mathrm{VF}_{2}$ in terms of additive $(1 / 2 \mathrm{D})$ and dominance $(1 / 4 \mathrm{H})$ are derived by Mather (1949). Heritability in both broad and narrow senses were determined by Powers et al. (1950) and Warner (1952), respectively. Expected and predicted values of genetic advance (GS and GS \%) were calculated according to Johnson et al. (1955). The phenotypic correlation coefficient was performed according to the procedure of Dewey and Lu (1959).

\section{RESULTS AND DISCUSSION 1- Means of the parents and their generation:}

The best source of information about the question of base on these estimates is that derived by fitting a model to the mean of the basic generation, i.e., $P_{1}, P_{2}, F_{1}, F_{2}, B C_{1}$ and $\mathrm{BC}_{2}$, which are presented in Tables 1 and 2 . The results revealed that wide range of means was recorded among the two parents in most of the studied traits under water deficit and normal conditions. The $F_{1}$ mean values were higher than the highest parent for root length, root volume, number of roots/ plant, root/ shoot ratio, hulling $\%$, milling $\%$, head rice $\%$ and amylose content $\%$ in both studied crosses under water deficit and normal conditions, and it was also higher than the highest parent for grain length in the cross I under water deficit and normal conditions.

Besides, the $F_{1}$ mean values were intermediate between the two parents for grain shape in the two crosses and grain length in the cross II (Giza $159 \times$ Takanari 1) under water deficit and normal conditions. On the other hand, the $F_{2}$ mean values were higher than the highest parent for root volume in the cross II and number of roots/ plant in the two crosses under water deficit and normal conditions, and both root/ shoot ratio and head rice \% in the cross II under normal condition, While for the other traits, $\mathrm{F}_{2}$ mean values were intermediate between the two parents, except hulling \% in both crosses under water deficit condition, head rice $\%$ in the cross II under water deficit condition was lower than the lowest parent. Moreover, $\mathrm{BC}_{1}$ mean values were higher than the highest parent for root length in the cross II under water deficit condition and in the cross I (Sakha 102 x IR 83142-B-60-B) under normal condition, root volume in the cross II under water deficit and the cross I under normal condition, number of roots/ plant in both crosses under water deficit and the crosses I and II under normal condition, root/ shoot ratio in the cross II, hulling $\%$ and 
head rice \% in the cross II under normal condition and milling \% in the cross II under water deficit condition. $\mathrm{BC}_{2}$ mean values were higher than the highest parent for root length, number of roots/ plant, root volume under normal conditions while were intermediate between the two parents for grain length in the cross I under normal condition, head rice \% in the cross II and amylose content $\%$ in the cross I under water deficit condition and the cross I under normal condition.

Table 1: Means and standard error of the six populations for rice root characters in the two studied crosses under water deficit (D) and normal (N) conditions.

\begin{tabular}{|c|c|c|c|c|c|c|c|c|}
\hline \multirow{2}{*}{ Characters } & \multirow{2}{*}{\multicolumn{2}{|c|}{ Cross }} & \multicolumn{6}{|c|}{ Mean performance and standard error } \\
\hline & & & $\mathrm{P}_{1}$ & $\mathrm{P}_{2}$ & $F_{1}$ & $\mathrm{BC}_{\mathrm{l}}$ & $\mathrm{BC}_{2}$ & $\mathrm{~F}_{2}$ \\
\hline \multirow{4}{*}{$\begin{array}{l}\text { Root length } \\
\quad(\mathrm{cm})\end{array}$} & \multirow{2}{*}{ I } & $\mathrm{D}$ & $21.23 \pm 0.17$ & $25.22 \pm 0.19$ & $28.43 \pm 0.23$ & $24.87 \pm 0.13$ & $25.9 \pm 0.12$ & $22.22 \pm 0.53$ \\
\hline & & $\mathrm{N}$ & $26.03 \pm 0.12$ & $29.83 \pm 0.11$ & $38.35 \pm 0.11$ & $29.90 \pm 0.11$ & $34.37 \pm 0.11$ & $27.58 \pm 0.35$ \\
\hline & \multirow{2}{*}{ II } & $\mathrm{D}$ & $18.87 \pm 0.17$ & $21.38 \pm 0.17$ & $25.81 \pm 0.20$ & $22.22 \pm 0.12$ & $22.52 \pm 0.14$ & $19.94 \pm 0.46$ \\
\hline & & $\mathrm{N}$ & $21.97 \pm 0.13$ & $26.78 \pm 0.12$ & $32.03 \pm 0.12$ & $25.92 \pm 0.11$ & $27.35 \pm 0.13$ & $24.30 \pm 0.29$ \\
\hline \multirow{4}{*}{$\begin{array}{l}\text { Root volume } \\
\qquad\left(\mathrm{cm}^{3}\right)\end{array}$} & \multirow{2}{*}{ I } & $\mathrm{D}$ & $40.52 \pm 0.53$ & $60.42 \pm 0.54$ & $106.2 \pm 0.72$ & $58.98 \pm 0.54$ & $65.5 \pm 0.37$ & $49.39 \pm 1.49$ \\
\hline & & $\mathrm{N}$ & $53.87 \pm 0.18$ & $80.87 \pm 0.14$ & $119.96 \pm 0.31$ & $84.51 \pm 0.32$ & $94.98 \pm 0.12$ & $68.67 \pm 1.11$ \\
\hline & \multirow{2}{*}{ II } & $\mathrm{D}$ & $19.40 \pm 0.35$ & $23.53 \pm 0.36$ & $75.05 \pm 0.64$ & $36.15 \pm 0.56$ & $39.98 \pm 0.61$ & $33.42 \pm 1.43$ \\
\hline & & $\mathrm{N}$ & 30.1 & $34.26 \pm 0.42$ & $93.82 \pm 0.26$ & $54.71 \pm 0.29$ & $71.85 \pm 0.30$ & $52.02 \pm 0.91$ \\
\hline \multirow{4}{*}{$\begin{array}{l}\text { Number of } \\
\text { roots/plant }\end{array}$} & \multirow{2}{*}{ I } & $\mathrm{D}$ & $25.97 \pm 0.76$ & $141.3 \pm 1.18$ & \pm 1.59 & $150.36 \pm 1.2$ & $162.78 \pm 1.3$ & $143.41 \pm 3.2$ \\
\hline & & $\mathrm{N}$ & $174.13 \pm 0.63$ & $242.7 \pm 0.47$ & $353.2 \pm 0.66$ & $244.1 \pm 0.60$ & $267.45 \pm 0.65$ & $244.02 \pm 2.65$ \\
\hline & \multirow{2}{*}{ II } & $\mathrm{D}$ & $96.60 \pm 1.20$ & $73.28 \pm 0.93$ & $192.81 \pm 2.0$ & $157.9 \pm 2.12$ & $150.25 \pm 1.7$ & $132.71 \pm 4.47$ \\
\hline & & $\mathrm{N}$ & $176.85 \pm 0.32$ & $123.4 \pm 0.18$ & $259.8 \pm 0.24$ & $213.8 \pm 0.60$ & $238.32 \pm 0.65$ & $205.05 \pm 2.10$ \\
\hline \multirow{4}{*}{$\begin{array}{c}\text { Root/Shoot } \\
\text { ratio }(\%)\end{array}$} & \multirow{2}{*}{ I } & $D$ & $0.36 \pm 0.01$ & $0.43 \pm 0.013$ & $0.60 \pm 0.01$ & $0.36 \pm 0.01$ & $0.48 \pm 0.01$ & $0.42 \pm 0.020$ \\
\hline & & $\mathrm{N}$ & $0.43 \pm 0.01$ & $0.78 \pm 0.01$ & $0.94 \pm 0.01$ & $0.53 \pm 0.01$ & $0.71 \pm 0.01$ & $0.71 \pm 0.02$ \\
\hline & \multirow{2}{*}{ II } & $D$ & $0.34 \pm 0.01$ & $0.31 \pm 0.01$ & $0.54 \pm 0.01$ & $0.51 \pm 0.10$ & $0.50 \pm 0.01$ & $0.40 \pm 0.020$ \\
\hline & & $\mathrm{N}$ & $0.49 \pm 0.01$ & $0.53 \pm 0.01$ & $0.88 \pm 0.01$ & $0.57 \pm 0.01$ & $0.70 \pm 0.01$ & $0.56 \pm 0.02$ \\
\hline
\end{tabular}

Crosses I: Sakha 102 x IR 83142-B-60-B, II: Giza 159x Takanari 1, D: water deficit, N: normal conditions.

Table 2: Means and standard errors of the six populations of grain quality characters for the two studied crosses under water deficit (D) and normal (N) conditions.

\begin{tabular}{|c|c|c|c|c|c|c|c|c|}
\hline \multirow{2}{*}{ characters } & \multirow{2}{*}{\multicolumn{2}{|c|}{ crosses }} & \multicolumn{6}{|c|}{ Mean performance and standard error } \\
\hline & & & $P_{1}$ & $\mathrm{P}_{2}$ & $\mathrm{~F}_{1}$ & $\mathrm{BC}_{1}$ & $\mathrm{BC}_{2}$ & $\mathrm{~F}_{2}$ \\
\hline \multirow{4}{*}{$\begin{array}{l}\text { Grain } \\
\text { length } \\
(\mathrm{mm})\end{array}$} & \multirow{2}{*}{1} & $\mathrm{D}$ & $7.59 \pm 0.013$ & $7.74 \pm 0.015$ & $7.83 \pm 0.01$ & $7.67 \pm 0.014$ & $7.70 \pm 0.012$ & $7.64 \pm 0.025$ \\
\hline & & $\mathrm{N}$ & $7.98 \pm 0.01$ & $8.49 \pm 0.01$ & $8.67 \pm 0.01$ & $8.09 \pm 0.01$ & $8.60 \pm 0.01$ & $8.44 \pm 0.03$ \\
\hline & \multirow{2}{*}{ II } & $\mathrm{D}$ & $6.98 \pm 0.016$ & $9.96 \pm 0.015$ & $8.33 \pm 0.014$ & $7.55 \pm 0.013$ & $8.30 \pm 0.011$ & $7.61 \pm 0.03$ \\
\hline & & $\mathrm{N}$ & $7.90 \pm 0.01$ & $10.80 \pm 0.01$ & $9.05 \pm 0.01$ & $8.55 \pm 0.01$ & $8.99 \pm 0.01$ & $8.45 \pm 0.03$ \\
\hline \multirow{4}{*}{$\begin{array}{l}\text { Grain } \\
\text { shape } \\
(\mathrm{mm})\end{array}$} & \multirow[b]{2}{*}{1} & $\mathrm{D}$ & $2.16 \pm 0.01$ & $2.88 \pm 0.012$ & $2.39 \pm 0.01$ & $2.44 \pm 0.014$ & $2.73 \pm 0.015$ & $2.27 \pm 0.027$ \\
\hline & & $\mathrm{N}$ & $2.21 \pm 0.01$ & $2.87 \pm 0.01$ & $2.38 \pm 0.01$ & $2.65 \pm 0.01$ & $2.59 \pm 0.01$ & $2.28 \pm 0.02$ \\
\hline & & $\mathrm{D}$ & $2.12 \pm 0.01$ & $3.30 \pm 0.01$ & $2.51 \pm 0.011$ & $2.18 \pm 0.01$ & $2.48 \pm 0.012$ & $2.44 \pm 0.026$ \\
\hline & & $\mathrm{N}$ & $2.20 \pm 0.01$ & $3.32 \pm 0.01$ & $2.55 \pm 0.01$ & $2.18 \pm 0.01$ & $2.66 \pm 0.01$ & $2.41 \pm 0.02$ \\
\hline
\end{tabular}

Crosses I: Sakha 102 x IR 83142-B-60-B, II: Giza 159x Takanari 1, D: water deficit, N: normal conditions. 
Table 2: Cont.

\begin{tabular}{|c|c|c|c|c|c|c|c|c|}
\hline \multirow{2}{*}{ characters } & \multirow{2}{*}{\multicolumn{2}{|c|}{ crosses }} & \multicolumn{6}{|c|}{ Mean performance and standard error } \\
\hline & & & $\mathrm{P}_{1}$ & $\mathrm{P}_{2}$ & $\mathrm{~F}_{1}$ & $\mathrm{BC}_{1}$ & $\mathrm{BC}_{2}$ & $\mathrm{~F}_{2}$ \\
\hline \multirow{4}{*}{$\begin{array}{l}\text { Hulling } \\
\text { (\%) }\end{array}$} & \multirow{2}{*}{ I } & $\mathrm{D}$ & $80.08 \pm 0.12$ & $79.80 \pm 0.11$ & $82.31 \pm 0.12$ & $78.53 \pm 0.13$ & $79.91 \pm 0.22$ & $78.39 \pm 0.48$ \\
\hline & & $\mathrm{N}$ & $83.91 \pm 0.14$ & $81.93 \pm 0.13$ & $85.01 \pm 0.13$ & $83.88 \pm 0.14$ & $82.08 \pm 0.13$ & $83.29 \pm 0.33$ \\
\hline & \multirow{2}{*}{ II } & $\mathrm{D}$ & $79.07 \pm 0.13$ & $77.87 \pm 0.12$ & $80.42 \pm 0.13$ & $78.95 \pm 0.13$ & $78.36 \pm 0.12$ & $77.45 \pm 0.29$ \\
\hline & & $\mathrm{N}$ & $83.41 \pm 0.11$ & $81.78 \pm 0.13$ & $84.93 \pm 0.14$ & $83.6 \pm 20.13$ & $82.28 \pm 0.13$ & $82.94 \pm 0.33$ \\
\hline \multirow{4}{*}{ Milling (\%) } & \multirow{2}{*}{1} & $\mathrm{D}$ & $69.88 \pm 0.11$ & $66.87 \pm 0.12$ & $71.00 \pm 0.13$ & $68.98 \pm 0.12$ & $68.33 \pm 0.11$ & $67.80 \pm 0.33$ \\
\hline & & $\mathrm{N}$ & $72.16 \pm 0.12$ & $69.76 \pm 0.12$ & $72.53 \pm 0.11$ & $69.57 \pm 0.11$ & $71.00 \pm 0.13$ & $70.61 \pm 0.35$ \\
\hline & \multirow{2}{*}{ II } & $\mathrm{D}$ & $68.95 \pm 0.14$ & $67.02 \pm 0.12$ & $70.18 \pm 0.11$ & $68.58 \pm 0.12$ & $68.30 \pm 0.12$ & $67.86 \pm 0.33$ \\
\hline & & $\mathrm{N}$ & $71.13 \pm 0.12$ & $69.92 \pm 0.11$ & $71.97 \pm 0.12$ & $70.01 \pm 0.12$ & $70.23 \pm 0.14$ & $69.50 \pm 0.33$ \\
\hline \multirow{4}{*}{$\begin{array}{l}\text { Head rice } \\
(\%)\end{array}$} & \multirow{2}{*}{1} & $\mathrm{D}$ & $62.02 \pm 0.10$ & $57.03 \pm 0.12$ & $63.05 \pm 0.12$ & $59.08 \pm 0.12$ & $58.40 \pm 0.11$ & $59.77 \pm 0.32$ \\
\hline & & $\mathrm{N}$ & $62.93 \pm 0.12$ & $59.80 \pm 0.11$ & $63.40 \pm 0.13$ & $61.01 \pm 0.13$ & $60.30 \pm 0.12$ & $61.97 \pm 0.32$ \\
\hline & \multirow{2}{*}{ II } & $\mathrm{D}$ & $60.10 \pm 0.04$ & $60.90 \pm 0.03$ & $62.27 \pm 0.12$ & $60.21 \pm 0.12$ & $61.02 \pm 0.11$ & $59.56 \pm 0.29$ \\
\hline & & $\mathrm{N}$ & $62.06 \pm 0.13$ & $62.28 \pm 0.16$ & $63.70 \pm 0.15$ & $62.40 \pm 0.13$ & $62.95 \pm 0.14$ & $62.60 \pm 0.37$ \\
\hline \multirow{4}{*}{$\begin{array}{c}\text { Amylose } \\
\text { content } \\
(\%)\end{array}$} & \multirow{2}{*}{1} & $\mathrm{D}$ & $21.02 \pm 0.12$ & $25.32 \pm 0.14$ & $29.00 \pm 0.12$ & $23.37 \pm 0.10$ & $25.51 \pm 0.11$ & $24.89 \pm 0.33$ \\
\hline & & $\mathrm{N}$ & $19.67 \pm 0.13$ & $24.31 \pm 0.13$ & $25.47 \pm 0.12$ & $23.81 \pm 0.11$ & $24.47 \pm 0.12$ & $23.72 \pm 0.30$ \\
\hline & \multirow{2}{*}{ II } & $\mathrm{D}$ & $20.98 \pm 0.12$ & $25.02 \pm 0.14$ & $28.72 \pm 0.12$ & $22.67 \pm 0.12$ & $24.02 \pm 0.13$ & $24.60 \pm 0.30$ \\
\hline & & $\mathrm{N}$ & $19.41 \pm 0.13$ & $24.13 \pm 0.15$ & $25.05 \pm 0.12$ & $22.07 \pm 0.12$ & $23.03 \pm 0.13$ & $23.55 \pm 0.30$ \\
\hline
\end{tabular}

Crosses I: Sakha 102 x IR 83142-B-60-B, II: Giza 159x Takanari 1, D: water deficit, N: normal conditions.

\section{2- Estimates of heterosis and degree of dominance:}

As shown in Tables 3 and 4 the degree of dominance was greater than unity $( \pm 1.0)$ for root length, root volume, number of roots/ plant, root/ shoot ratio, hulling \%, milling \%, head rice \% and amylose content in both crosses and the cross I for grain length under water deficiency and normal conditions, suggesting the importance of over-dominance in controlling these traits. However, the degrees of dominance were lesser than unity for grain shape in both crosses and grain length in the cross II under water deficit and normal conditions. The ratios which were between zero and unity, suggesting partial or incomplete dominance and might play a remarkable role in the inheritance of these traits. The same results were previously obtained by Abd-Allah (2000), Abd El- Lattef and Mady (2009), El-Abd et al. (2008), Hijam et al. (2012) and Ravikumar et al. (2014).
It is clear in Tables 3 and 4 that significant and highly significant and positive estimates of heterosis as a deviation from mid- and better-parents were obtained for root length, root volume, number of roots/plant, root/shoot ratio, hulling $\%$, Milling $\%$, head rice $\%$ and amylose content $\%$ in both crosses, while for grain length in cross I under both water deficit and normal conditions.

While for the other remaining traits, i.e. grain length in cross II and grain shape in both crosses exhibited highly significant negative heterosis as a deviation from midparents under both water deficit and normal conditions. Similar results were reported earlier by Abd El-Lattef et al. (2008), Ganapathy and Ganesh (2008), Abd-Allah (2009), Hassan et al. (2011), Mall et al. (2011), Concepcion et al. (2015) and Guimaraes et al. (2016). 
Table 3: Estimates of heterosis as a deviation from mid-parents (MP), better-parent (BP) and degree of dominance of rice root characters, for the two studied crosses under water deficit $(\mathrm{D})$ and normal $(\mathrm{N})$ conditions.

\begin{tabular}{|c|c|c|c|c|c|c|c|}
\hline \multirow{3}{*}{ Characters } & \multirow{3}{*}{ Cr. } & \multicolumn{4}{|c|}{ Heterosis \% } & \multirow{3}{*}{$\begin{array}{c}\text { Degree of } \\
\text { dominance } \\
(\mathrm{N})\end{array}$} & \multirow{3}{*}{$\begin{array}{l}\text { Degree of } \\
\text { dominance } \\
\text { (D) }\end{array}$} \\
\hline & & \multicolumn{2}{|c|}{ MP } & \multicolumn{2}{|c|}{$\mathrm{BP}$} & & \\
\hline & & $\mathrm{N}$ & $D$ & $\mathrm{~N}$ & D & & \\
\hline \multirow{2}{*}{$\begin{array}{l}\text { Root length } \\
\quad(\mathrm{cm})\end{array}$} & I & $22.41^{\star *}$ & $37.27^{\star *}$ & $12.73^{\star *}$ & $28.52^{\star *}$ & -14.26 & -20.18 \\
\hline & II & $28.22^{\star \star}$ & $31.40^{* *}$ & $20.68^{\star *}$ & $19.59^{\star *}$ & 4.52 & 3.18 \\
\hline \multirow{2}{*}{$\begin{array}{l}\text { Root volume } \\
\qquad\left(\mathrm{cm}^{3}\right)\end{array}$} & 1 & $110.57^{\star *}$ & $78.05^{\star \star}$ & $75.89^{\star \star}$ & $48.33^{* *}$ & -5.6 & -3.89 \\
\hline & II & $249.57^{\star *}$ & $191.38^{\star *}$ & $218.85^{\star \star}$ & $173.84^{\star \star}$ & -25.9 & -29.87 \\
\hline \multirow{2}{*}{$\begin{array}{l}\text { Number of } \\
\text { roots/ plant }\end{array}$} & 1 & $45.54^{\star \star}$ & $69.50^{\star *}$ & $37.65^{\star \star}$ & $45.56^{\star \star}$ & -7.94 & -4.22 \\
\hline & II & $126.98^{\star *}$ & $73.08^{\star *}$ & $99.59^{\star \star}$ & $46.92^{\star *}$ & 9.25 & 4.1 \\
\hline \multirow{2}{*}{$\begin{array}{c}\text { Root/ Shoot } \\
\text { ratio (\%) }\end{array}$} & I & $51.06^{\star *}$ & $54.35^{\star \star}$ & $38.77^{\star \star}$ & $20.40^{\star \star}$ & -5.76 & -1.92 \\
\hline & II & $68.75^{\star \star}$ & $71.79^{* *}$ & $58.82^{\star *}$ & $64.99^{* *}$ & 16.03 & -17.41 \\
\hline
\end{tabular}

${ }^{*},{ }^{* *}$ : Significant and highly significant at 0.05 and 0.01 levels of probability, respectively.

Crosses I: Sakha 102x IR 83142-B-60-B, II: Giza 159 x Takanari 1, D: water deficit, N: normal conditions.

Table 4: Estimates of heterosis as a deviation from mid-parents (MP), better-parent (BP) and degree of dominance of rice grain quality characters, for the two studied crosses under water deficit (D) and normal (N) conditions.

\begin{tabular}{|c|c|c|c|c|c|c|c|}
\hline \multirow{3}{*}{ Characters } & \multirow{3}{*}{ Cr. } & \multicolumn{4}{|c|}{ Heterosis \% } & \multirow{3}{*}{$\begin{array}{c}\text { Degree of } \\
\text { dominance } \\
(\mathrm{N})\end{array}$} & \multirow{3}{*}{$\begin{array}{c}\text { Degree of } \\
\text { dominance } \\
\text { (D) }\end{array}$} \\
\hline & & \multicolumn{2}{|c|}{ MP } & \multicolumn{2}{|c|}{$\mathrm{BP}$} & & \\
\hline & & $\mathrm{N}$ & D & $N$ & $\mathrm{D}$ & & \\
\hline \multirow{2}{*}{$\begin{array}{l}\text { Grain length } \\
\quad(\mathrm{mm})\end{array}$} & I & $2.22^{\star \star}$ & $5.38^{* *}$ & $3.29^{* *}$ & $8.75^{\star \star}$ & -2.14 & -1.73 \\
\hline & II & $-1.65^{\star \star}$ & $-3.15^{\star *}$ & $19.33^{\star \star}$ & $14.59^{* *}$ & 0.09 & 0.2 \\
\hline \multirow{2}{*}{$\begin{array}{l}\text { Grain shape } \\
(\mathrm{mm})\end{array}$} & 1 & $-5.20^{* *}$ & $-6.54^{* *}$ & $10.52^{\star *}$ & $7.43^{\star \star}$ & 0.36 & 0.5 \\
\hline & II & $-7.19^{\star \star}$ & $-7.83^{\star *}$ & $18.58^{\star \star}$ & $15.60^{\star *}$ & 0.33 & 0.38 \\
\hline \multirow{2}{*}{ Hulling (\%) } & 1 & $2.96^{\star *}$ & $2.51^{* *}$ & $2.77^{\star *}$ & $1.31^{* *}$ & 16.47 & 2.11 \\
\hline & II & $2.48^{\star \star}$ & $2.82^{\star \star}$ & $1.70^{\star \star}$ & $1.82^{\star *}$ & 3.25 & 2.87 \\
\hline \multirow{2}{*}{ Milling (\%) } & 1 & $3.82^{* \star}$ & $2.21^{* *}$ & $1.59^{\star *}$ & 0.51 & 1.73 & 1.31 \\
\hline & II & $3.23^{* *}$ & $2.04^{* *}$ & $1.79^{\star \star}$ & $1.17^{* *}$ & 2.28 & 2.38 \\
\hline \multirow{2}{*}{ Head rice (\%) } & 1 & $5.91^{\star \star}$ & $3.30^{* *}$ & $1.65^{\star *}$ & 0.73 & 1.41 & 1.29 \\
\hline & II & $2.90^{* \star}$ & $2.45^{\star \star}$ & $2.21^{\star *}$ & $2.26^{\star *}$ & -4.26 & -13.55 \\
\hline \multirow{2}{*}{$\begin{array}{c}\text { Amylose } \\
\text { content (\%) }\end{array}$} & 1 & $25.13^{* *}$ & $15.82^{* *}$ & $37.93^{* *}$ & $29.47^{\star *}$ & -2.7 & -1.5 \\
\hline & II & $24.85^{\star *}$ & $15.04^{* *}$ & $36.86^{* *}$ & $29.04^{\star \star}$ & -2.83 & -1.38 \\
\hline
\end{tabular}

*, **: Significant and highly significant at 0.05 and 0.01 levels of probability, respectively.

Crosses I: Sakha 102 x IR 83142-B-60-B, II: Giza 159xTakanari 1, D: water deficit, N: normal conditions. 


\section{3- Estimates components mean:}

As shown in Tables 5 and 6 that mean effect parameter $(m)$ was highly significant for all the studied root and grain quality traits. Additive gene action (d) played an important role in the inheritance of all the studied characters, except root length and root/ shoot ratio in the cross II under water deficit condition, grain length in the cross I under water deficit condition and milling \% in the cross II under both water deficit and normal conditions. Moreover, dominance gene action (h) played a greater role in the inheritance of all the studied traits in both crosses, except root/ shoot ratio and grain length, hulling $\%$ and milling $\%$ in the cross I under normal condition and in the cross II under normal condition for grain shape, hulling $\%$, head rice $\%$ and amylose content $\%$ in the cross II under normal condition.

Table 5: Genetic components of generation means of rice root characters for the two studied crosses under water deficiency (D) and normal (N) conditions.

\begin{tabular}{|c|c|c|c|c|c|c|c|c|}
\hline \multirow{2}{*}{ Characters } & \multirow{2}{*}{\multicolumn{2}{|c|}{ Cross }} & \multicolumn{6}{|c|}{ Genetic components of generation mean } \\
\hline & & & $\mathrm{m}$ & d & $\mathrm{h}$ & $\mathrm{i}$ & $\mathrm{J}$ & I \\
\hline \multirow{4}{*}{$\begin{array}{l}\text { Root length } \\
\quad(\mathrm{cm})\end{array}$} & \multirow{2}{*}{1} & D & $22.22^{* *}$ & $-1.02^{* *}$ & $17.85^{\star *}$ & $12.65^{\star *}$ & $0.96^{* *}$ & $-10.86^{\star *}$ \\
\hline & & $\mathrm{N}$ & $27.58^{* *}$ & $-4.47^{\star *}$ & $28.62^{\star *}$ & $18.21^{* *}$ & $-2.57^{\star *}$ & $-14.19^{\star *}$ \\
\hline & \multirow{2}{*}{ II } & D & $19.94^{\star *}$ & -0.30 & $15.41^{* *}$ & $9.73^{\star *}$ & $0.95^{\star \star}$ & $-7.34^{* *}$ \\
\hline & & $\mathrm{N}$ & $24.33^{\star *}$ & $-1.42^{\star *}$ & $17.00^{* *}$ & $9.35^{\star *}$ & $0.98^{* \star}$ & $-3.06^{*}$ \\
\hline \multirow{4}{*}{$\begin{array}{l}\text { Root volume } \\
\qquad\left(\mathrm{cm}^{3}\right)\end{array}$} & \multirow{2}{*}{ I } & D & $49.39 * *$ & $-6.51^{* *}$ & $107.22^{\star \star}$ & $51.40^{\star *}$ & $3.43^{\star \star}$ & 13.14 \\
\hline & & $\mathrm{N}$ & $68.67^{* \star}$ & $-10.47^{\star *}$ & $136.88^{* *}$ & $84.30^{\star *}$ & $3.02^{* *}$ & $-68.62^{\star *}$ \\
\hline & \multirow{2}{*}{ II } & D & $33.42^{* *}$ & $-3.83^{\star \star}$ & $72.15^{\star *}$ & $18.57^{* *}$ & $-1.76^{*}$ & $22.18^{* *}$ \\
\hline & & $\mathrm{N}$ & $52.02^{* *}$ & $-17.13^{* *}$ & $106.65^{* *}$ & $45.02^{* *}$ & $-15.07^{\star \star}$ & $-46.10^{* *}$ \\
\hline \multirow{4}{*}{$\begin{array}{l}\text { Number of } \\
\text { roots/ plant }\end{array}$} & \multirow{2}{*}{1} & D & $143.41^{* *}$ & $-12.42^{* *}$ & $113.49^{* *}$ & $52.63^{\star *}$ & $-4.76^{\star}$ & -22.65 \\
\hline & & $\mathrm{N}$ & $244.02^{\star \star}$ & $-23.27^{\star *}$ & $192.01^{* *}$ & $47.15^{\star *}$ & $11.00^{* *}$ & $53.01^{* *}$ \\
\hline & \multirow{2}{*}{ II } & D & $132.71^{* *}$ & $7.65^{\star *}$ & $193.31^{* *}$ & $85.45^{\star *}$ & -4.00 & $-146.42^{\star *}$ \\
\hline & & $\mathrm{N}$ & $205.05^{* *}$ & $-24.52^{\star *}$ & $193.72^{\star *}$ & $84.01^{* *}$ & $-51.25^{\star *}$ & $-168.34^{\star *}$ \\
\hline \multirow{4}{*}{$\begin{array}{c}\text { Root/ Shoot } \\
\text { ratio (\%) }\end{array}$} & \multirow{2}{*}{ I } & D & $0.42^{* *}$ & $0.11^{* *}$ & $0.22^{*}$ & 0.022 & $-0.08^{* *}$ & 0.27 \\
\hline & & $\mathrm{N}$ & $0.71^{* *}$ & $-0.17^{\star \star}$ & -0.02 & -0.35 & 0.004 & $0.96^{* *}$ \\
\hline & \multirow{2}{*}{ II } & D & $0.40^{* *}$ & 0.012 & $0.62^{* *}$ & $0.41^{* *}$ & -0.0002 & $-0.72^{* *}$ \\
\hline & & $\mathrm{N}$ & $0.56^{* *}$ & $-0.12^{* *}$ & $0.67^{* *}$ & $0.30^{* *}$ & $-0.1^{* *}$ & -0.06 \\
\hline
\end{tabular}

m: mid-parent value.

$\mathrm{d}$ and $\mathrm{h}$ : pooled additive and dominance effects, respectively.

$\mathrm{i}, \mathrm{j}$ and $\mathrm{I}$ : pooled additive $\mathrm{x}$ additive, additive $\mathrm{x}$ dominance and dominance $\mathrm{x}$ dominance gene interaction, respectively.

${ }^{*},{ }^{* *}$ : Significant and highly significant at 0.05 and 0.01 levels of probability, respectively.

Crosses I: Sakha 102 x IR 83142-B-60-B, II: Giza 159xTakanari 1, D: water deficit, N: normal conditions. 
Table 6: Genetic components of generation means of rice grain quality characters for the two studied crosses under water deficiency (D) and normal (N) conditions.

\begin{tabular}{|c|c|c|c|c|c|c|c|c|}
\hline \multirow{2}{*}{ Characters } & \multirow{2}{*}{\multicolumn{2}{|c|}{ Cross }} & \multicolumn{6}{|c|}{ Genetic components of generation mean } \\
\hline & & & \multirow{2}{*}{$\frac{m}{7.64^{* *}}$} & \multirow{2}{*}{$\frac{D}{-0.03}$} & \multirow{2}{*}{$\frac{h}{0.34^{\star *}}$} & \multirow{2}{*}{$\frac{i}{0.17}$} & \multirow{2}{*}{$\frac{j}{0.044^{*}}$} & \multirow{2}{*}{$\frac{1}{0.09}$} \\
\hline \multirow{4}{*}{ Grain length (mm) } & \multirow{2}{*}{ I } & D & & & & & & \\
\hline & & $\mathrm{N}$ & $8.44^{\star *}$ & $-0.50^{* *}$ & 0.07 & $-0.37^{* *}$ & $-0.25^{\star *}$ & $0.80^{\star \star}$ \\
\hline & \multirow{2}{*}{ II } & D & $7.61^{\star \star}$ & $-0.74^{* *}$ & $1.13^{\star \star}$ & $1.27^{\star \star}$ & $0.74^{\star \star}$ & $0.62^{* \star}$ \\
\hline & & $\mathrm{N}$ & $8.45^{\star *}$ & $-0.43^{* *}$ & $0.95^{\star *}$ & $1.24^{\star *}$ & $1.01^{* *}$ & $0.48^{\star \star}$ \\
\hline \multirow{4}{*}{ Grain shape (mm) } & \multirow{2}{*}{ I } & D & $2.27^{\star \star}$ & $-0.28^{* *}$ & $1.14^{\star \star}$ & $1.27^{\star *}$ & $0.07^{\star *}$ & $-1.77^{\star *}$ \\
\hline & & $\mathrm{N}$ & $2.28^{* \star}$ & $0.05^{\star *}$ & $1.20^{\star \star}$ & $1.30^{\star *}$ & $0.39^{* \star}$ & $-2.01^{* *}$ \\
\hline & & D & $2.44^{\star *}$ & $-0.29^{* *}$ & $-0.61^{* *}$ & $-0.41^{* *}$ & $0.29^{\star \star}$ & $1.53^{\star \star}$ \\
\hline & $\|$ & $\mathrm{N}$ & $2.41^{* *}$ & $-0.48^{* *}$ & -0.18 & 0.02 & $0.07^{\star *}$ & $0.90^{\star \star}$ \\
\hline \multirow{4}{*}{ Hulling (\%) } & \multirow{2}{*}{ I } & D & $78.39^{\star *}$ & $-1.37^{* *}$ & $5.70^{\star \star}$ & 3.33 & $-1.51^{\star *}$ & 4.27 \\
\hline & & $N$ & $83.29^{\star \star}$ & $1.80^{* \star}$ & 0.87 & -1.31 & $0.81^{* *}$ & $5.14^{\star \star}$ \\
\hline & 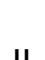 & D & $77.45^{\star *}$ & $0.58^{* *}$ & $6.77^{\star *}$ & $4.82^{* *}$ & -0.01 & -1.65 \\
\hline & 11 & $N$ & $82.94^{\star *}$ & $1.33^{* *}$ & 2.39 & 2.46 & $0.52^{*}$ & $3.19^{*}$ \\
\hline \multirow{4}{*}{ Milling (\%) } & \multirow{2}{*}{1} & D & $67.80^{* *}$ & $0.65^{\star *}$ & $6.06^{* *}$ & $3.45^{\star}$ & $-0.85^{\star *}$ & 0.66 \\
\hline & & $\mathrm{N}$ & $70.61^{* *}$ & $-1.42^{* *}$ & 0.25 & -1.31 & $-2.62^{* *}$ & $7.16^{* \star}$ \\
\hline & 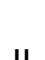 & D & $67.86^{* *}$ & 0.28 & $4.50^{* *}$ & 2.30 & $-0.67^{* *}$ & 0.26 \\
\hline & 11 & $N$ & $69.50^{\star *}$ & -0.22 & $3.91^{* *}$ & 2.46 & $-0.83^{* *}$ & 2.04 \\
\hline \multirow{4}{*}{ Head rice (\%) } & \multirow{2}{*}{1} & D & $59.77^{* *}$ & $0.68^{* *}$ & -0.60 & $-4.12^{\star *}$ & $-1.80^{* *}$ & $14.31^{* *}$ \\
\hline & & $N$ & $61.97^{\star \star}$ & $0.71^{\star \star}$ & $-3.24^{*}$ & $-5.27^{\star *}$ & $-0.85^{\star *}$ & $12.18^{\star \star}$ \\
\hline & 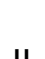 & D & $59.65^{\star *}$ & $-0.81^{* *}$ & $5.63^{* *}$ & $3.87^{* *}$ & $-0.39^{*}$ & -0.77 \\
\hline & " & $\mathrm{N}$ & $62.60^{* *}$ & $-0.55^{\star *}$ & 1.82 & 0.30 & -0.43 & 0.75 \\
\hline \multirow{4}{*}{$\begin{array}{l}\text { Amylose content } \\
(\%)\end{array}$} & \multirow{2}{*}{ I } & D & $24.89^{* *}$ & $-2.13^{\star *}$ & $4.03^{* *}$ & -1.79 & 0.01 & $8.36^{* *}$ \\
\hline & & $\mathrm{N}$ & $23.72^{* *}$ & $-0.66^{\star *}$ & $5.15^{\star *}$ & 1.67 & $1.65^{\star *}$ & $-3.31^{*}$ \\
\hline & \multirow{2}{*}{ II } & D & $24.60^{* *}$ & $-1.35^{\star *}$ & 0.68 & $-5.03^{* *}$ & $0.66^{* *}$ & $15.09^{* *}$ \\
\hline & & $\mathrm{N}$ & $23.55^{\star *}$ & $-0.96^{\star *}$ & -0.73 & $-4.00^{\star *}$ & $1.40^{* *}$ & $7.43^{\star *}$ \\
\hline
\end{tabular}

m: mid-parent value.

$\mathrm{d}$ and $\mathrm{h}$ : pooled additive and dominance effects, respectively.

$\mathrm{i}, \mathrm{j}$ and $\mathrm{I}$ : pooled additive $\mathrm{x}$ additive, additive $\mathrm{x}$ dominance and dominance $\mathrm{x}$ dominance gene interaction, respectively.

${ }^{*},{ }^{* \star}$ : Significant and highly significant at 0.05 and 0.01 levels of probability, respectively.

Crosses I: Sakha 102 x IR 83142-B-60-B, II: Giza 159xTakanari 1, D: water deficit, N: normal conditions.

Additive $\mathrm{x}$ additive type of gene interaction (i) had played an effective role in the inheritance of all traits in the two crosses, except root/ shoot ratio in the cross I under both water deficit and normal conditions, grain length in the cross I under 
water deficiency condition, grain shape in the cross II under normal condition, hulling $\%$ in the cross I under water deficiency and normal conditions and the cross II under normal condition, milling \% in the cross II under both water deficit and normal conditions and the cross I under normal condition, head rice $\%$ in the cross II under normal condition and amylose content $\%$ in the cross I under water deficit and normal conditions. These findings suggest that additive gene effects made a significant contribution to the inheritance of the studied characters in these crosses. Additive gene effects can be exploited in early generations because the dominance effects were also non-significant and lower in magnitude than these additive effects. Similar results were reported by Shehata et al. (2004), Manickavelu et al. (2006), Kumar et al. (2006), El-Abd et al. (2008), Hassan et al. (2011), Hijam et al. (2012), Ravikumar et al. (2014) and Guimaraes et al. (2016).

Additive $x$ dominance type of gene interaction (j) played an important role in the inheritance of all the studied characters, except number of roots/ plant in the cross II under water deficit condition, root/ shoot ratio in the cross II under water deficit condition and the cross I under normal condition, hulling $\%$ in the cross II under water deficit conditions and head rice $\%$ in the cross II under normal condition. Dominance $x$ dominance type of gene interaction (I) had played an important role in the inheritance of all the studied characters, except root volume and number of roots/ plant in the cross I under water deficit condition and root / shoot ratio in the cross II under normal condition and the cross I under water deficit condition, grain length in the cross I under water deficit condition, hulling $\%$ in cross I under water deficit conditions, milling \% in the cross II under water deficit and normal conditions and the cross I under water deficit condition, head rice \% in the cross II under both water deficit and normal conditions. In contrast, dominance gene action; additive $\mathrm{x}$ dominance and dominance $x$ dominance type of gene interaction showed highly significant values, indicating that these factors are significant contributors to the variation of generation means and played an important role in the inheritance of such characters. Since additive gene effects were insignificant for these characters, simple selection procedure in the early generations may not contribute significantly to the improvement of these characters. The additive components in these traits can be successfully exploited through pedigree method of selection because of major contribution of additive gene effects in late generations of segregating populations. These results were in agreement with those obtained previously by Shehata et al. (2004), Manickavelu et al. (2006), Kumar et al. (2006), El-Abd et al. (2008), Hassan et al. (2011), Hijam et al. (2012), Ravikumar et al. (2014) and Guimaraes et al. (2016).

\section{4- Estimates of genetic variance, heritability and genetic advance:}

Data summarized in Tables 7 and 8 revealed that additive genetic variance $(1 / 2 D)$ was higher than dominance genetic variance $(1 / 4 \mathrm{H})$ for all the studied characters under water deficiency and normal conditions, indicating that additive component of genetic variance was predominant in the expression for all the studied characters. Heritability in broad sense estimates were larger than their corresponding ones of narrow sense heritability for all the studied crosses. High broad sense heritability and high genetic advance were estimated for some root and grain quality characters. Narrow sense heritability ranged from low to moderate in both studied crosses. Similar results were reported by Toorchi et al. (2002), Gomez and Kalamani (2003), Abd El-Lattef et al. (2008) Hijam et al. (2012) and Concepcion et al. (2015). 
Table 7: Estimates of additive genetic variance $(1 / 2 D)$, dominance genetic variance $(1 / 4 H)$, broad and narrow-sense heritabilities and genetic advance (G.S \%) of rice root characters for the two studied crosses under water deficiency (D) and normal (N) conditions.

\begin{tabular}{|c|c|c|c|c|c|c|c|c|}
\hline \multirow[b]{2}{*}{ Characters } & \multirow{2}{*}{\multicolumn{2}{|c|}{ Cross }} & \multicolumn{2}{|c|}{ Genetic variance } & \multicolumn{2}{|c|}{ Heritability \% } & \multirow[b]{2}{*}{ G.S } & \multirow[b]{2}{*}{ G.S \% } \\
\hline & & & $1 / 2 D$ & $1 / 4 \mathrm{H}$ & $\begin{array}{l}\text { Broad- } \\
\text { sense }\end{array}$ & $\begin{array}{l}\text { Narrow- } \\
\text { sense }\end{array}$ & & \\
\hline \multirow{4}{*}{ Root length (cm) } & \multirow{2}{*}{ I } & $\mathrm{D}$ & 0.53 & -0.29 & 85.32 & 12.20 & 13.42 & 60.38 \\
\hline & & $\mathrm{N}$ & 0.22 & -0.11 & 89.01 & 22.06 & 16.09 & 58.35 \\
\hline & \multirow{2}{*}{ II } & D & 0.39 & -0.21 & 83.59 & 15.89 & 15.21 & 76.27 \\
\hline & & $\mathrm{N}$ & 0.13 & -0.06 & 81.15 & 38.34 & 23.02 & 94.75 \\
\hline \multirow{4}{*}{ Root volume $\left(\mathrm{cm}^{3}\right)$} & \multirow{2}{*}{ I } & $D$ & 4.03 & -2.16 & 83.38 & 19.50 & 60.04 & 121.57 \\
\hline & & $\mathrm{N}$ & 2.38 & -1.18 & 95.95 & 9.58 & 22.09 & 32.17 \\
\hline & \multirow{2}{*}{ II } & $\mathrm{D}$ & 3.43 & -1.59 & 89.09 & 33.68 & 99.78 & 298.51 \\
\hline & & $\mathrm{N}$ & 1.50 & -0.77 & 86.41 & 21.46 & 40.65 & 78.14 \\
\hline \multirow{4}{*}{$\begin{array}{l}\text { Number of } \\
\text { roots/plant }\end{array}$} & \multirow{2}{*}{ I } & $\mathrm{D}$ & 18.23 & -8.98 & 85.92 & 30.69 & 207.52 & 144.69 \\
\hline & & $\mathrm{N}$ & 13.28 & -6.60 & 94.91 & 11.27 & 61.61 & 25.24 \\
\hline & \multirow{2}{*}{ II } & $\mathrm{D}$ & 32.47 & -14.68 & 88.84 & 37.79 & 348.03 & 262.52 \\
\hline & & $\mathrm{N}$ & 8.05 & -3.70 & 98.48 & 17.6 & 76.24 & 37.18 \\
\hline \multirow{4}{*}{$\begin{array}{c}\text { Root/ Shoot ratio } \\
(\%)\end{array}$} & \multirow{2}{*}{ I } & $\mathrm{D}$ & 0.001 & -0.0006 & 71.20 & 31.32 & 1.67 & 399.12 \\
\hline & & $\mathrm{N}$ & 0.0009 & -0.0005 & 60.46 & 42.33 & 2.09 & 293.84 \\
\hline & \multirow{2}{*}{ II } & $D$ & 0.0008 & -0.0004 & 80.36 & 39.31 & 1.91 & 469.39 \\
\hline & & $\mathrm{N}$ & 0.0008 & -0.0004 & 71.49 & 47.25 & 2.36 & 418.65 \\
\hline
\end{tabular}

Crosses I: Sakha 102 x IR 83142-B-60-B, II: Giza 159xTakanari 1, D: water deficit, N: normal conditions.

Table 8: Estimates of additive genetic variance (1/2 D), dominance genetic variance (1/4 $H$ ), broad and narrow-sense heritabilities and genetic advance (G.S \%) of rice grain quality characters for the two studied crosses under water deficiency (D) and normal $(\mathrm{N})$ conditions.

\begin{tabular}{|c|c|c|c|c|c|c|c|c|}
\hline \multirow{2}{*}{ Characters } & & & \multicolumn{2}{|c|}{ Genetic variance } & \multicolumn{2}{|c|}{ Heritability \% } & \multirow[b]{2}{*}{ G.S } & \multirow[b]{2}{*}{ G.S \% } \\
\hline & \multicolumn{2}{|c|}{ Cross } & $1 / 2 D$ & $1 / 4 \mathrm{H}$ & $\begin{array}{l}\text { Broad- } \\
\text { sense }\end{array}$ & $\begin{array}{l}\text { Narrow- } \\
\text { sense }\end{array}$ & & \\
\hline \multirow{4}{*}{ Grain length (mm) } & \multirow{2}{*}{ I } & D & 0.0009 & -0.0004 & 72.27 & 59.18 & 3.08 & 40.35 \\
\hline & & $\mathrm{N}$ & 0.002 & -0.001 & 87.36 & 24.93 & 1.80 & 21.37 \\
\hline & \multirow{2}{*}{ II } & $\mathrm{D}$ & 0.001 & -0.001 & 77.91 & 28.05 & 1.93 & 25.38 \\
\hline & & $\mathrm{N}$ & 0.002 & -0.001 & 86.43 & 27.17 & 1.98 & 23.45 \\
\hline \multirow{4}{*}{ Grain shape (mm) } & \multirow{2}{*}{1} & $D$ & 0.001 & -0.0004 & 83.31 & 59.11 & 3.30 & 145.51 \\
\hline & & $\mathrm{N}$ & 0.001 & -0.0006 & 80.89 & 34.63 & 2.02 & 88.64 \\
\hline & \multirow{2}{*}{ II } & $D$ & 0.001 & -0.0005 & 82.90 & 37.84 & 2.04 & 83.69 \\
\hline & & $\mathrm{N}$ & 0.0008 & -0.0004 & 76.03 & 51.96 & 2.54 & 105.11 \\
\hline
\end{tabular}

Crosses I: Sakha 102 x IR 83142-B-60-B, II: Giza 159xTakanari 1, D: water deficit, N: normal conditions. 
Table 8: Cont.

\begin{tabular}{|c|c|c|c|c|c|c|c|c|}
\hline \multirow{2}{*}{ Characters } & & & \multicolumn{2}{|c|}{ Genetic variance } & \multicolumn{2}{|c|}{ Heritability \% } & \multirow[b]{2}{*}{ G.S } & \multirow[b]{2}{*}{ G.S \% } \\
\hline & \multicolumn{2}{|c|}{ Cross } & $1 / 2 \mathrm{D}$ & $1 / 4 \mathrm{H}$ & Broad- & Narrow- & & \\
\hline \multirow{4}{*}{ Hulling (\%) } & \multirow{2}{*}{1} & D & 0.40 & -0.18 & 93.52 & 29.89 & 29.91 & 38.16 \\
\hline & & $\mathrm{N}$ & 0.18 & -0.09 & 83.03 & 34.23 & 23.68 & 28.43 \\
\hline & \multirow{2}{*}{ II } & D & 0.14 & -0.07 & 80.07 & 36.49 & 22.15 & 28.59 \\
\hline & & $\mathrm{N}$ & 0.18 & -0.09 & 84.37 & 32.99 & 22.59 & 27.24 \\
\hline \multirow{4}{*}{ Milling (\%) } & \multirow{2}{*}{ I } & $\mathrm{D}$ & 0.19 & -0.09 & 93.52 & 26.70 & 18.31 & 27.00 \\
\hline & & $\mathrm{N}$ & 0.22 & -0.11 & 88.44 & 25.31 & 18.68 & 26.46 \\
\hline & \multirow{2}{*}{ II } & $\mathrm{D}$ & 0.18 & -0.09 & 80.07 & 28.99 & 19.87 & 29.29 \\
\hline & & $\mathrm{N}$ & 0.19 & -0.09 & 87.08 & 31.82 & 22.12 & 31.83 \\
\hline \multirow{4}{*}{ Head rice (\%) } & \multirow{2}{*}{ I } & $\mathrm{D}$ & 0.18 & -0.09 & 86.20 & 25.95 & 17.44 & 29.19 \\
\hline & & $\mathrm{N}$ & 0.17 & -0.08 & 84.51 & 33.25 & 21.97 & 35.45 \\
\hline & & $\mathrm{D}$ & 0.14 & -0.06 & 92.56 & 33.25 & 20.01 & 33.55 \\
\hline & & $\mathrm{N}$ & 0.23 & -0.11 & 83.69 & 29.89 & 22.79 & 36.41 \\
\hline \multirow{4}{*}{$\begin{array}{c}\text { Amylose content } \\
(\%)\end{array}$} & \multirow{2}{*}{ I } & $\mathrm{D}$ & 0.19 & -0.1 & 84.35 & 22.40 & 15.32 & 61.57 \\
\hline & & $\mathrm{N}$ & 0.15 & -0.08 & 80.74 & 30.15 & 19.00 & 80.09 \\
\hline & \multirow{2}{*}{ II } & $\mathrm{D}$ & 0.15 & -0.07 & 81.83 & 36.23 & 22.69 & 92.24 \\
\hline & & $\mathrm{N}$ & 0.14 & -0.07 & 78.77 & 37.45 & 23.42 & 99.43 \\
\hline
\end{tabular}

Crosses I: Sakha 102 x IR 83142-B-60-B, II: Giza 159xTakanari 1, D: water deficit, N: normal conditions.

\section{5- Estimates of phenotypic correlation coefficients:}

The phenotypic correlation coefficients among all possible pairs of grain yield component traits are presented in Tables 9 and10.

Lucidly, grain yield was positively and strongly correlated with each of root length, root volume, number of roots/ plant, root/ shoot ratio, grain length, grain shape, hulling $\%$, milling $\%$ and head rice $\%$ in the two studied crosses under both water deficiency and normal conditions. Therefore, any selection based on these traits will bring the desired improvement in grain yield. Amylose content showed insignificant negative and positive correlation with most other grain quality traits. Root length was highly significant and positive associated with root volume, number of roots/ plant and root/ shoot ratio in the studied crosses. However, a highly significant and positive estimate of phenotypic correlation coefficient was recorded between grain length and grain shape, hulling $\%$, milling $\%$ and head rice $\%$. Present findings coincide with the results of Abd El-Lattef and Mady (2009), 
Hassan et al. (2011) and Zulqarnain et al. (2012).

Therefore, any selection based on these traits will bring the desired improvement in grain yield. Amylose content showed insignificant negative and positive correlation with most other grain quality traits. Root length was highly significant and positive associated with root volume, number of roots/ plant and root/ shoot ratio in both studied crosses. However, a highly significant and positive estimate of phenotypic correlation coefficient was recorded between grain length and grain shape, hulling $\%$, milling $\%$ and head rice $\%$. Present findings coincide with the results of Abd El-Lattef and mady (2009), Hassan et al. (2011) and Zulqarnain et al. (2012).

Table 9: Phenotypic correlation coefficient among all possible pairs of root characters in the $F_{2}$ generation of the crosses I and II under water deficiency (D) and normal (N) conditions.

\begin{tabular}{|c|c|c|c|c|c|c|}
\hline Characters & \multicolumn{2}{|c|}{ Cross } & 1 & 2 & 3 & 4 \\
\hline \multirow{4}{*}{ 1- Root length (cm) } & \multirow{2}{*}{ I } & $\mathrm{D}$ & & & & \\
\hline & & $\mathrm{N}$ & & & & \\
\hline & \multirow{2}{*}{ II } & $\mathrm{D}$ & & & & \\
\hline & & $\mathrm{N}$ & & & & \\
\hline \multirow{4}{*}{ 2- Root volume $\left(\mathrm{cm}^{3}\right)$} & \multirow{2}{*}{1} & $\mathrm{D}$ & $0.818^{\star *}$ & & & \\
\hline & & $\mathrm{N}$ & $0.73^{\star *}$ & & & \\
\hline & \multirow{2}{*}{ II } & $\mathrm{D}$ & $0.775^{\star \star}$ & & & \\
\hline & & $\mathrm{N}$ & $0.81^{* *}$ & & & \\
\hline \multirow{4}{*}{ 3- Number of roots/plant } & \multirow{2}{*}{1} & $\mathrm{D}$ & $0.818^{\star *}$ & $0.943^{* *}$ & & \\
\hline & & $\mathrm{N}$ & $0.82^{* *}$ & $0.84^{* *}$ & & \\
\hline & \multirow{2}{*}{ II } & $\mathrm{D}$ & $0.795^{\star *}$ & $0.949^{* *}$ & & \\
\hline & & $\mathrm{N}$ & $0.67^{* *}$ & $0.84^{* *}$ & & \\
\hline \multirow{4}{*}{ 4- Root/ shoot ratio } & \multirow{2}{*}{ I } & $\mathrm{D}$ & $0.943^{* *}$ & $0.846^{* *}$ & $0.852^{* *}$ & \\
\hline & & $\mathrm{N}$ & $0.74^{* *}$ & $0.73^{* *}$ & $0.86^{* *}$ & \\
\hline & 11 & $\mathrm{D}$ & $0.409^{* *}$ & $0.632^{* *}$ & $0.627^{\star *}$ & \\
\hline & 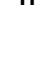 & $\mathrm{N}$ & $0.64^{* *}$ & $0.79^{* *}$ & $0.72^{* *}$ & \\
\hline \multirow{4}{*}{ 5- Grain yield/plant (g) } & \multirow{2}{*}{1} & $\mathrm{D}$ & $0.936^{\star \star}$ & $0.863^{\star *}$ & $0.879^{\star *}$ & $0.953^{\star *}$ \\
\hline & & $\mathrm{N}$ & $0.82^{* *}$ & $0.82^{* *}$ & $0.94^{* *}$ & $0.84^{* *}$ \\
\hline & \multirow{2}{*}{ II } & $\mathrm{D}$ & $0.674^{\star \star}$ & $0.857^{\star *}$ & $0.863^{\star *}$ & $0.724^{\star \star}$ \\
\hline & & $\mathrm{N}$ & $0.65^{\star *}$ & $0.80^{* *}$ & $0.75^{\star *}$ & $0.75^{\star *}$ \\
\hline
\end{tabular}

*, ** Significant and highly significant at 0.05 and 0.01 levels of probability, respectively.

Crosses I: Sakha 102 x IR 83142-B-60-B, II: Giza 159xTakanari 1, D: water deficit, N: normal conditions. 
Table 10: Phenotypic correlation coefficient among all possible pairs of grain quality characters in the $F_{2}$ generation of the crosses I and II under water deficiency (D) and normal $(\mathrm{N})$ conditions.

\begin{tabular}{|c|c|c|c|c|c|c|c|c|}
\hline Characters & \multicolumn{2}{|c|}{ Cross } & 1 & 2 & 3 & 4 & 5 & 6 \\
\hline \multirow{4}{*}{ 1- Grain length (mm) } & \multirow{2}{*}{1} & $D$ & & & & & & \\
\hline & & $\mathrm{N}$ & & & & & & \\
\hline & \multirow{2}{*}{ II } & $\mathrm{D}$ & & & & & & \\
\hline & & $\mathrm{N}$ & & & & & & \\
\hline \multirow{4}{*}{ 2- Grain shape } & \multirow{2}{*}{1} & $D$ & $0.564^{* *}$ & & & & & \\
\hline & & $\mathrm{N}$ & $0.62^{* \star}$ & & & & & \\
\hline & $\mu$ & $D$ & $0.782^{* *}$ & & & & & \\
\hline & 11 & $\mathrm{~N}$ & 0.02 & & & & & \\
\hline \multirow{4}{*}{ 3- Hulling (\%) } & \multirow{2}{*}{1} & $D$ & $0.751^{* *}$ & $0.537^{* *}$ & & & & \\
\hline & & $\mathrm{N}$ & $0.92^{\star \star}$ & $0.57^{\star \star}$ & & & & \\
\hline & $\mu$ & $D$ & $0.841^{* *}$ & $0.733^{* *}$ & & & & \\
\hline & $\pi$ & $\mathrm{N}$ & $0.74^{* *}$ & 0.06 & & & & \\
\hline \multirow{4}{*}{ 4- Milling (\%) } & \multirow{2}{*}{1} & $D$ & $0.798^{* *}$ & $0.567^{* *}$ & $0.691^{* *}$ & & & \\
\hline & & $\mathrm{N}$ & $0.82^{* *}$ & $0.55^{\star *}$ & $0.83^{* *}$ & & & \\
\hline & \multirow{2}{*}{ II } & $D$ & $0.759^{* *}$ & $0.732^{* *}$ & $0.748^{* *}$ & & & \\
\hline & & $\mathrm{N}$ & $0.82^{\star \star}$ & 0.03 & $0.78^{\star \star}$ & & & \\
\hline \multirow{4}{*}{ 5- Head rice (\%) } & \multirow{2}{*}{1} & $D$ & $0.814^{* *}$ & $0.578^{* *}$ & $0.672^{* *}$ & $0.736^{* *}$ & & \\
\hline & & $\mathrm{N}$ & $0.81^{* *}$ & $0.56^{* *}$ & $0.85^{\star *}$ & $0.74^{\star \star}$ & & \\
\hline & \multirow{2}{*}{ II } & $D$ & $0.620^{* *}$ & $0.617^{* *}$ & $0.675^{* *}$ & $0.583^{* *}$ & & \\
\hline & & $\mathrm{N}$ & $0.74^{* *}$ & 0.05 & $0.69^{* *}$ & $0.71^{* *}$ & & \\
\hline \multirow{4}{*}{$\begin{array}{l}\text { 6- Amylose content } \\
(\%)\end{array}$} & \multirow{2}{*}{ I } & $\mathrm{D}$ & 0.029 & -0.125 & -0.057 & -0.067 & 0.048 & \\
\hline & & $\mathrm{N}$ & $-0.32^{*}$ & $-0.36^{* *}$ & $-0.29^{\star}$ & $-0.32^{*}$ & $-0.33^{*}$ & \\
\hline & \multirow{2}{*}{ II } & $\mathrm{D}$ & 0.114 & 0.071 & -0.015 & 0.086 & -0.038 & \\
\hline & & $N$ & $-0.27^{*}$ & 0.14 & $-0.27^{\star}$ & -0.25 & $-0.35^{*}$ & \\
\hline \multirow{4}{*}{$\begin{array}{l}\text { 7- Grain yield/plant } \\
\text { (g) }\end{array}$} & \multirow{2}{*}{ I } & $D$ & $0.950^{* *}$ & $0.581^{* *}$ & $0.794^{* *}$ & $0.810^{* *}$ & $0.846^{* *}$ & -0.002 \\
\hline & & $\mathrm{N}$ & $0.91^{* *}$ & $0.59^{* *}$ & $0.92^{* *}$ & $0.81^{* *}$ & $0.83^{\star *}$ & $-0.29^{*}$ \\
\hline & II & $D$ & $0.948^{* *}$ & $0.820^{* *}$ & $0.880^{* *}$ & $0.779^{* *}$ & $677^{\star *}$ & 0.068 \\
\hline & & $N$ & $0.84^{\star *}$ & 0.08 & $0.74^{\star \star}$ & $0.79^{\star \star}$ & $0.72^{\star \star}$ & $-0.30^{*}$ \\
\hline
\end{tabular}

${ }^{*},{ }^{* *}$ Significant and highly significant at 0.05 and 0.01 levels of probability, respectively.

Crosses I: Sakha 102 x IR 83142-B-60-B, II: Giza 159xTakanari 1, D: water deficit, N: normal conditions. 


\section{REFERANCES}

Abd Allah, A. A. (2009). Genetic studies on leaf rolling and some root traits under drought conditions in rice (Oryza sativa L.). African J. of Biotechnology, 8 (22): 6241-6248.

Abd Allah, A.A. (2000). Breeding studies on rice (Oryza sativa L.). Ph.D. Thesis, Fac. Agric. Menoufiya Univ., Shibin El-Kom, Egypt.

Abd El-Lattef, A. S. and A. A. Mady (2009). Genetic behavior for some root characters and their relation to some other characters under drought condition in rice (Oryza sativa L.). J. Agric. Sci. Mansoura. Univ., 34(2): 1153-1172.

Abd El-Lattef, A. S. M., A. B. El- Abd, A. A. Madyan and W. M. H. El- Khouby (2008). Inheritance of earliness, grain yield and some grain quality traits in rice (Oryza sativa L.). Under water deficiency conditions. J. Agric. Res. Kafrelsheikh Univ., 34(4): 993-1019.

Boualaphanh, C., M. Calingacion, R.P. Cuevas, D. Jothityangkoon, J. Sanitchon, M. Fitzgerald, F. Wei, H. Tao, S. Lin and B. Bouman (2011). Yield and quality of traditional and improved Lao varieties of rice. Scienceasia 37: 89-97.

Brar, D.S. and G.S. Khush (2013). Biotechnological approaches for increasing productivity and sustainability of rice production. In: Bhullar, G.S., Bhullar, N.K. (Eds.), Agricultural Sustainability. Academic Press, San Diego, CA, pp. 151-175 (Chapter 8).

Butany, W. T. (1961). Mass emasculation in rice. Inster. Rice Comm. Newsletter, 9: 913.

Concepcion, J.C., M. Ouk, D. Zhao and M. A. Fitzgerald (2015). The need for new tools and investment to improve the accuracy of selecting for grain quality in rice. Field Crops Research, 182: 60 - 67.

Dewey, D. R. and K. H. Lu (1959). A correlation and path-coefficient analysis of components of crested wheat grass seed production. Agron. J. 51: $515-518$.
EL-Abd, A. B., S. E. M. Sedeek, S. A. A. Hammoud and A. A. Abd Allah (2008). Studies on genetic variability, heritability and genetic advance for grain yield and grain quality traits in some promising genotypes of rice (Oryza sativa L.). J. Agric. Res., Kafrelsheikh Univ., 34(1): 7397.

Falconer, D. S. and F.C. Mackey (1996). Introduction to quantitative genetics. Fourth Edition. Longman. New York.

Ganapathy, S. and S. K. Ganesh (2008). Heterosis analysis for physioMorphological traits in relation to drought tolerance in rice (Oryza sativa, L.). World J. of Agric. Sci., 4 (5): 623-629.

Gomez, S. M and A. Kalamani (2003). Scope of landraces for future drought tolerance breeding programme in rice (Oryza sativa L.). Plant-Archives, 3(1): 77-79.

Guimaraes, C.M., L.F. Stone and A.C.D. Silva (2016). Evapotranspiration and grain yield of upland rice as affected by water deficit. Rev. Bras. Eng. Agric. Ambient, 20(5): 441-446.

Hassan, H. M., A. B. El-Abd and N. M. ElBaghdady (2011). Combining ability for some root, physiological and grain quality traits in rice (Oryza Sativa L.) under water deficit conditions. J. Agric. Res. Kafrelsheikh Univ., 37(2): 239-256.

Hayman, B. I. (1958). The separation of epistatic from additive and dominance variation means. Heredity, 12: 371-390.

Hijam, L., S. Das Dewanjee and K. K. Sarkar (2012). Influence of root characters on drought resistance in rice (Oryza sativa L.). Indian Journal of Genetics and Plant Breeding. 72 (2): 221 $-225$.

Inthapanya, P., C. Boualaphanh and Schiller J.M. Hatsadong (2006). The history of lowland rice variety improvements in Laos. In: Schiller, J.M., Chanphengxay, M.B., Linquist, B., Rao, S.A. (Eds.), Rice in Laos. International Rice Research Institute, Manila, pp. 325-358. 
Jodon, N. E. (1938). Experiment on artificial hybridization of rice. J. Amer. Soci. Argon., 30: 249-305.

Johnson, H. W., H. F. Robinson and R. E. Commstock (1955). Estimates of genetic and environmental variability in soybean. Agron. J. 47: 214-222.

Kumar, S., A. S. Gautam and S. Chandel (2006) Estimates of genetic parameters for quality traits in rice (Oryza sativa L.) in Mid Hills of Himachal Pradesh. Crop Research Hisar, 32(2): 206-208.

Mackill, D., B. Collard, C. Neeraja, R. Rodriguez, S. Heuer and A. Ismail (2006). QTLs in rice breeding: examples for abiotic stresses. In: Rice Genetics 5: Proceedings of the International Rice Genetics Symposium. International Rice Research Institute, Manila, pp. 155-167.

Mall, A.K., P. Swain, S. Das, O.N. Singh and A. Kumar (2011). Effect of drought on yield and drought susceptibility index for quality characters of promising rice genotypes. Cereal Research Communications, 39(1):22-31.

Manickavelu, A., N. Nadarajan, S. K. Ganesh and R. P. Gnanamalar (2006). Genetic analysis of biparental progenies in rice (Oryza sativa L.). Asian J. of Plant Sci., 5(1): 33-36.

Mather, K. (1949). Biometrical Genetics. Dover Publication, inc. London.

Mather, K. and J.I. Jinks (1971). Biometrical Genetics. Cornell Univ. Press Ithaca, N.Y., 231pp.

Pandey, S., H. Bhandari, R. Sharan, D. Naik, S. K. Taunk and A. D. Sastri (2005). Economic costs of drought and rainfed rice farmers' coping mechanisms in eastern India. Final Project Report. IRRI, Los Bañ os, Philippines.

Powers, L. R., L. F. Locke and J. C. Garrett (1950). Partitioning method of genetic analysis applied to quantitative characters of tomato crosses. U. S. Dept. Agr. Tech. bull., 998: 56 pp.
R.R.T.C. (Rice Research and Training center) (2013). The Eight National Rice Research and Development Program Workshop. RRTC., ARC., Egypt. pp. 34.

Ravikumar, G., P. Manimaran, S.R. Voleti, D. Subrahmanyam, R.M. Sundaram, K.C. Bansal, B.C. Viraktamath and S.M. Balachandran (2014). Stress-inducible expression of AtDREB1A transcription factor greatly improves drought stress tolerance in transgenic indica rice. Transgenic Res. 23: 421-439.

Shehata, S. M., A. E. Draz, A. A. Abd-Allah and B. A. Zayed (2004). Genetic studies on morphological characters as indicators of salt and drought tolerance in rice. Egypt. J. Agric. Res., 82 (1): 101118.

Singh, R.K., U.S. Singh and G.S. Khush (2000). Aromatic rices. Oxford \& IBH Publishing Co. Pvt Ltd., New Delhi, India.

Tomita, M. (2009). Introgression of Green Revolution sd1 gene into isogenic genome of rice super cultivar Koshihikari to create novel semidwarf cultivar 'Hikarishinseiki' (Koshihikari-sd1). Field Crop. Res. 114: 173-181.

Toorchi, M., H. E. Shashidhar1, S. Hittalmani and T. M. Gireesha (2002). Rice root morphology under contrasting moisture regimes and contribution of molecular marker heterozygosity. Euphytica, 126:251-25.

Warner, J. N (1952). A method for estimating heritability. Agron. J., 44: 427430.

Wynne, J. C., D. A. Emery and P. W. Rice (1970). Combining ability estimates in Arachis hypogea L. II- Field performance of $F_{1}$ hybrids. Crop Sci., 10: 713-715.

Zulqarnain, H., A. S. Khan and Z. Samta (2012). Correlation and path coefficient analysis of yield components in rice (Oryza sativa L.) under simulated drought stress condition. American-Eurasian J. of Agric. \& Environ. Sci., 12 (1): 100-104. 


\section{السلوك الوراثي لبعض صفات الجذر وصفات جودة الحبوب فى الأرز تحت الظروف الطيعية وندرة المياة}

حماده محمد حسن

قسم بحوث الارز - معهد بحوث المحاصيل الحقلية - مركز البحوث الزراعية - مصر

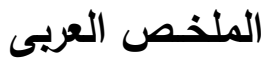

أجريت هذه التجربة بالمزرعة البحثية بمحة بحوث الارز - سخا - كفرالثيخ - مصـر وذلك خلال مواسم زراعة الأرز 2014، 2015 و2016 وذلك بهدف دراسة السلوك الوراثى بعض صفات الجذر وصفات جودة الحبوب فى الأرز تحت ظروف ندرة المياة وذلك باستخدام العشائر الستة (الأب الأول، الأب الثنانى، الجيل الأول، الجيل الثانى، الهجين الرجعى

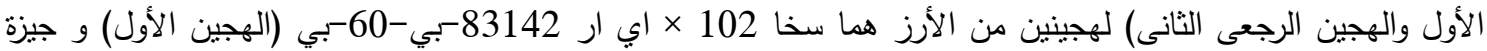

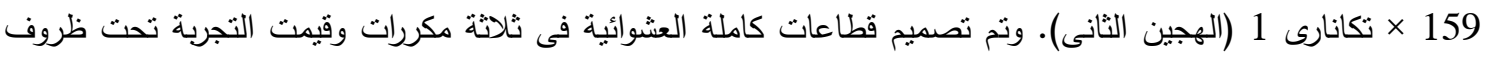
ندرة المياه وذلك باستخدام الرى السطى كل 12 يوم وكذلك تحت الظروف الطبيعية وكان الرى فيها كل أربعة أيام، ونت

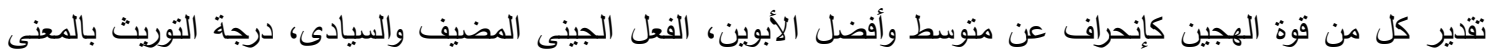
العريض والضيق، التحسين المنوقع من الإتنخاب وكذلك معامل الإرتباط المظهرى بين جميع الأزواج الممكنة لبعض صفات الجذر وصفات جودة الحبوب (طول الجذر، حجم الجذر، عدد الجذور/ نبات، نسبة الوزن الجاف للمجموع الجذرى إلى الإنى

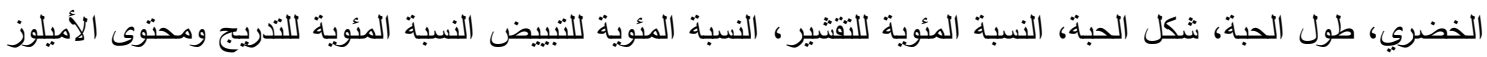
في الحبة. وأثشارت النتائج المتحصل عليها من الأباء والجيل الأول والأجيال الإنعزالية إلى وجود ددى واسع بين منوسطات القيم

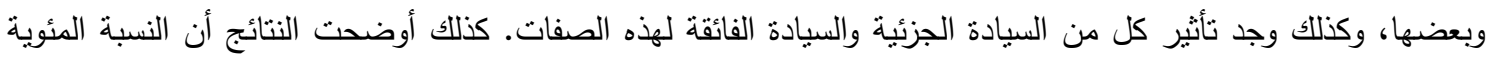

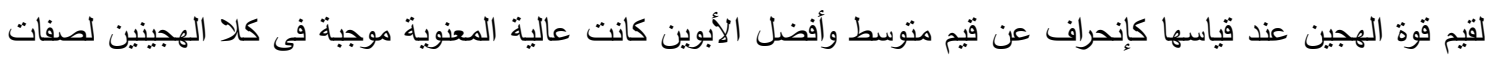
طول الجذر، حجم الجذر، عدد الجذور/ نبات، نسبة الوزن الجاف للمجموع الجذرى إلى الخضرى، النسبة المئوية للتقشير، التئية

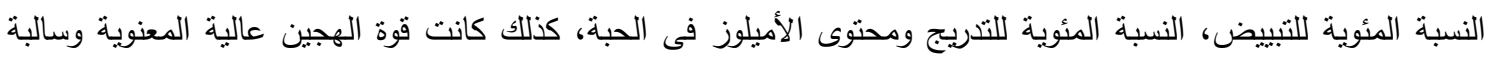
كإنحراف عن قيم متوسط الأبوين لصفات شكل الحبة فى كلا الهجينين وطول الحبة فى الهجين الثانى.

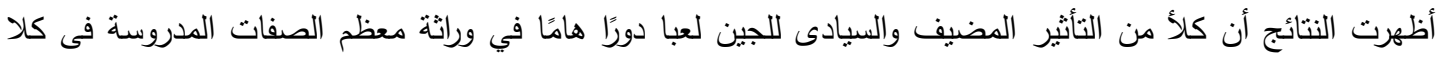
الهجينين. كذلك لعب كل من الفعل الجينى المضيف × المضيف ، المضيف × السيادى والسيادى × السيادى دورًا هامًا فى وراثة هذه الصفات عدا بعض الاسنثناءات.

أوضحت النتائج أن درجة التوريث بالمعنى الواسع كانت عالية لكل الهجن فى كل الصفات المدروسة ماعدا الهجين رقم

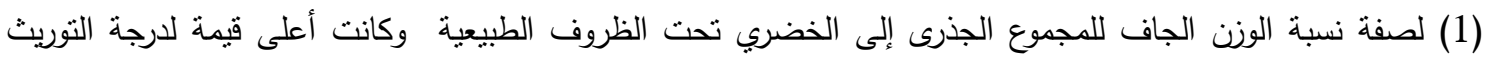

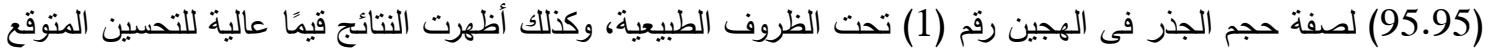
بالإنتخاب لمعظم الصفات المدروسة تحت كلتا الظروف لذلك يوصى بالإنتخاب المباثر فى الأجيال المبكرة لهذة الصفات،

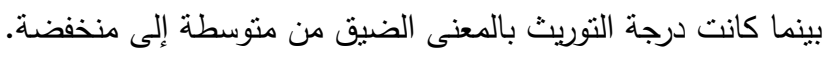

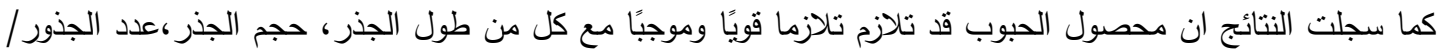

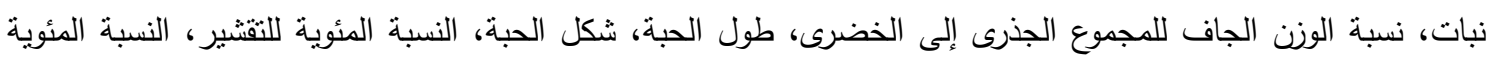

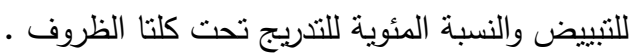


Genetic behavior of some root and grain quality traits under water

توصى الدراسة بأن الهجين جيزه 159 × تكانارى1 هو أفضل الهجن التي نستطيع أن نوصى به للنمو تحت ظروف ندرة

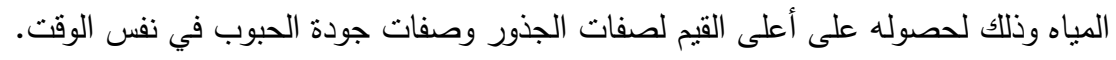

